\title{
DER MYTHOS EINER VERLORENEN HEIMAT - GALIZIEN. DEUTSCH-POLNISCHER GEDÄCHTNISRAUM IN DEN ROMANEN RADETZKYMARSCH VON JOSEPH ROTH UND DAS SALZ DER ERDE VON JOSEPH WITTLIN
}

Ostgalizien - Provinz am nordöstlichen Rand der untergegangenen österreichischungarischen Monarchie und multilingualer Vielvölkerstaat - nimmt in der unter anderem von der Literatur getragenen Erinnerungskultur ${ }^{1}$ einen festen Platz ein. Mit dem Zusammenbruch des Vielvölkerstaates 1918 aus dem allgemeinen Blickfeld gerückt und später noch aufgrund der politischen Veränderungen nach dem Zweiten Weltkrieg nicht nur als topographische Region, sondern auch als multikulturelle Landschaft von der Karte Europas verschwunden, erlebt es doch immer wieder seine Auferstehung: Es ist die Erinnerung an die Orte und Menschen sowie an das Leben zur Zeit der Habsburgermonarchie, die Galizien dem Vergessen entreißt. Hier setzt die Erinnerungsarbeit des Dichters, des Schriftstellers ein. Literarisch aufgearbeitete verlorene Orte und vergangene historische Ereignisse schaffen eine Wirklichkeit, die aus dem subjektiven Erleben und Erinnern des Autors entworfen, von Mythen umwobene Gedächtnisräume darbietet. ${ }^{2}$ Mythisiert wird von den

${ }^{1}$ „Erinnerungskultur beruht weitgehend, wenn auch keineswegs ausschließlich, auf Formen des Bezugs auf die Vergangenheit. (...) In der Erinnerung wird Vergangenheit rekonstruiert. In diesem Sinne ist die These gemeint, daß Vergangenheit dadurch entsteht, daß man sich auf sie bezieht." Siehe dazu Jan A s s m a n n : Das kulturelle Gedächtnis. München 1999, S. 31. - Die hier zu untersuchenden Romane beziehen sich auf das Vergangene (die Zeit der Donaumonarchie), sie beleben es und halten durch Rekonstruktion einen Teil des kulturellen Gedächtnisses fest. Vgl. dazu ebenda, S. 35 .

${ }^{2}$ Es entsteht der Eindruck, daß Radetzkymarsch einen Beitrag zum kulturellen Gedächtnis leistet, das „sich auf Fixpunkte in der Vergangenheit richte“, indem der Autor an die historische Figur (des Kaisers) sowie topographische und temporale Räume (die Zeit der Donaumonarchie und Galizien) erinnert und ihnen eine mythische Kraft verleiht. Assmann konstatiert über das Wechselspiel Erinnerungsfiguren-Mythos folgendermaßen: „Mythos ist eine fundierende Geschichte, eine Geschichte, die erzählt wird, um eine Gegenwart vom Ursprung her zu erhellen. (...) Durch Erinnerung 
Autoren das Leben in Galizien, und nur am Rande erwähnt man die dort herrschenden Sozialverhältnisse. Als irrelevant erweist sich für die Literatur auch die mit dem Mangel an ideologischer Homogenität dieser Region zusammenhängende Identitätskrise ihrer Bevölkerung, die aus der Vielfalt der Religionen, die in dem Vielvölkerstaat existieren, resultiert. Nicht $\mathrm{zu}$ übersehen ist auch der Mangel an gemeinsamer Geschichte, der bei der Bevölkerung Galiziens eine Identitätskrise auslöste.

Der Ausbruch des Ersten Weltkriegs erfüllte die Bevölkerung Galiziens eher mit Angst um ihre Zukunft als mit Hoffnung auf eine Änderung der Mißstände, in denen sie lebte. ${ }^{3}$ Galiziens Zusammenbruch hinterläßt bei seinen Bewohnern ein Gefühl des Verlustes. So steigt Galizien allmählich zu einem Mythos einer heilen Welt, einer verlorenen Heimat auf. ${ }^{4}$

Den Raum für die Mythenbildung und -dekonstruktion öffnet hier die Literatur. Joseph Roth hebt in Radetzkymarsch einerseits wohl unbestritten diesen Mythos hervor, doch andererseits läßt er „das Anachronistisch-Atavistische der Monarchie ${ }^{{ }^{5}}$ anklingen, womit er den Untergang dieser mythischen Welt für unumgänglich erklärt und ankündigt.

Eine kritische Auseinandersetzung mit der scheinbar heilen Welt der habsburgischen Provinz Galizien suchte auch der polnische Autor Józef Wittlin in seinem Roman Das Salz der Erde. Es scheint, daß Wittlin in diesem Roman den Mythos Galizien durch den Protagonisten Piotr Niewiadomski dekonstruiert.

Was sagen die in den Romanen Radetzkymarsch und Das Salz der Erde enthaltenen Gedächtnisräume über das Vergangene aus? Welche Gemeinsamkeiten, Parallelen weisen sie auf? Es gibt gewiß viele Aspekte, die eine komparative Analyse der hier zu untersuchenden Texte und die Aufschlüsselung des Hauptthemas ermöglichen würden. Vier Faktoren sollen eine Basis für den Versuch sein, Wittlins Das Salz der Erde mit Roths Radetzkymarsch zu vergleichen: die galizische Herkunft der Autoren beider Werke, die Thematik der Romane, der ideologische Hintergrund sowie auch die Gemeinsamkeiten, die sich auf der biographischen Ebene beider Autoren feststellen lassen.

Nur zwei Jahre trennen die Geburtsdaten von Roth (1894) und Wittlin (1896). Und nur 40 Kilometer voneinander entfernt liegen ihre galizischen Geburtsorte Brody und Dmytrów, nah an der Grenze, die zwei Kaiserreiche trennte. Die Schulzeit hat die Autoren noch nicht zusammengebracht. Erst zur Studienzeit in Lemberg, wo sie in derselben Straße gewohnt haben, und in Wien kreuzten sich ihre Wege. Selbst den Militärdienst haben sie gemeinsam abgeleistet. Ihre Wege trennten sich erst, als das Kaiserreich Franz Joseph I. zerfiel.

wird Geschichte zum Mythos. Dadurch wird sie nicht wirklich, sondern im Gegenteil erst Wirklichkeit im Sinne einer fortdauernden normativen und formativen Kraft." Ebenda, S. 52f.

${ }^{3} \mathrm{Zu}$ den Lebensbedingungen der galizischen Bevölkerung siehe zum Beispiel Stefan Kaszyński: Die Götterdämmerung in Galizien. Zur geschichtsmythologisierenden Rolle der Romane von Joseph Roth, Józef Wittlin und Julian Stryjkowski. In: Stefan K a s z y ń s k i (Hrsg.): Galizien - eine literarische Heimat. Poznań 1987, S. 55.

${ }^{4}$ Zum Galizien-Mythos vgl. auch: Ebenda, S. 55-64.

5 Adolf D. K 1 a r m a n n : Das Österreichbild im „Radetzkymarsch“. In: David B r o n s e n (Hrsg.): Joseph Roth und die Tradition. Agora Band 27, Darmstadt 1975, S. 154. 
Was die beiden Autoren verband, das waren ihre prägenden Eindrücke, die sie zur Aufarbeitung ähnlicher Themen und Motive in den erwähnten Romanen veranlaßten. Diese Eindrücke kreisen um die Begriffe Heimat, Heimatlosigkeit, Krieg und die Gestalt des Kaisers. Sie bilden die Grundlage der hier vorzunehmenden Analyse beider Romane.

Joseph Roth wurde 1884 in der ostgalizischen Stadt Brody in einer jüdischen Familie geboren. Von Brody aus konnte man die russische Grenze erreichen, wenn man die kaum 10 Kilometer lange Strecke zurücklegte. 800 Bahnkilometer trennten die Stadt von Wien, der Hauptstadt der Donaumonarchie. Brody, die Stadt der Kindheit des Autors, die Grenzstadt, taucht namenlos in Radetzkymarsch auf. Die Armut ihrer Bewohner und die mit den Zeichen des Verfalls versehene Landschaft kommen im Roman ins Bild:

\begin{abstract}
Die Händler jener Gegend lebten viel eher von Zufällen als von Aussichten, viel mehr von der unberechenbaren Vorsehung als von geschäftlichen Überlegungen, und jeder Händler war jederzeit bereit, die Ware zu ergreifen, die ihm das Schicksal jeweilig auslieferte, und auch eine Ware zu erfinden, wenn ihm Gott keine beschert hatte.(..) Sumpfgeborene waren die Menschen dieser Gegend. Denn die Sümpfe lagen unheimlich ausgebreitet über der ganzen Fläche des Landes, zu beiden Seiten der Landstraße, mit Fröschen, Fieberbazillen und tückischem Gras, das den ahnungslosen, des Landes unkundigen Wanderern eine furchtbare Lockung in einen furchtbaren Tod bedeutete. (...) Alle aber, die dort geboren waren, kannten die Tücke des Sumpfes und besaßen selbst etwas von seiner Tücke. (...) Wer immer von Fremden in diese Gegend geriet, mußte allmählich verlorengehn. Keiner war so kräftig wie der Sumpf. Niemand konnte der Grenze standhalten. (S. 94)
\end{abstract}

Die Grenzstadt Brody setzt im Roman einerseits die topographische Grenze der Monarchie, doch andererseits impliziert sie ihre temporale Grenze. Der Ort in Ostgalizien wird zum Schauplatz des unaufhaltsamen Untergangs der Donaumonarchie und auch seine Bewohner und Besucher werden an ihre existentielle Grenze versetzt, denn ,niemand konnte der Grenze standhalten“ (ebenda). ${ }^{7}$ Der Untergang der Donaumonarchie, dargestellt am Beispiel der verfallenden Familie von Trotta, bildet die Thematik des hier zu analysierenden Romans Radetzkymarsch.

Das Autobiographische, jene Grunderfahrung des Krieges und der Heimatlosigkeit - „Mein stärkstes Erlebnis war der Krieg und der Untergang meines Vaterlandes, des einzigen, das ich je besessen: die österreichisch-ungarische Monarchie", schreibt Roth in einem seiner Briefe ${ }^{8}-$, kommt im Radetzkymarsch

\footnotetext{
${ }^{6}$ Im folgenden wird zitiert aus Joseph R o th : Radetzkymarsch. Reinbek 1957.

${ }^{7}$ Martin P o 11 a c k verweist in seinem Buch Nach Galizien auf einen Spruch, dessen Bedeutung den oben erwähnten Zerfall mit einschließt: „In den achtziger Jahren des neunzehnten Jahrhunderts sagte man in Galizien gern verfallen wie in Brody, wenn man ausdrücken wollte, daß man von einem ungünstigen Geschick an einem düstern und traurigen Ort verschlagen worden war, von dem es kein Entrinnen gab; man war der Öde der morastigen Ebene anheimgefallen." Die andere Bedeutung bezieht sich auf die wirtschaftliche Lage der Grenzstadt und beinhaltete den Mißerfolg beim Schmuggeln, meist vom Tabak, über die Grenze. Beim Schmuggeln erwischt worden zu sein, hieß damals verfallen wie in Brody. Siehe Martin P o 11 a c k : Nach Galizien. Wien 1984, S. $185 f$.

${ }^{8}$ Aus Roths Brief vom 28.10.1932 an Prof. Otto Forst de Battagalia. In: Hermann K e s t e n (Hrsg.): Joseph Roth. Briefe 1911-1939. Köln/Berlin 1970, S. 240.
} 
zum Ausdruck. Seine Bindung an das verlorene Galizien formuliert Roth folgendermaßen: ,....weil meine Heimat nicht mehr vorhanden ist, bin ich nirgends zu Hause".

Thematisch läßt sich Roth im Radetzkymarsch als Dichter des Zerfalls erkennen. Sein Roman mutet an wie eine Elegie auf die glanzvolle Zeit, in der er gelebt hat, und die mit dem Zusammenbruch des kaiserlichen Imperiums $1918 \mathrm{zu}$ Ende ging. Den Zerfall der Monarchie und das Ende einer Weltordnung kündigt im Roman der polnische Graf Wojciech Chojnicki im Gespräch mit dem Bezirkshauptmann von Trotta an:

\begin{abstract}
„Wir alle leben nicht mehr!“ (...) Mit großer Anstrengung brachte Herr von Trotta noch die Frage zustande: „Ich verstehe nicht! Wie sollte die Monarchie nicht mehr dasein?" „Natürlich!“ erwiderte Chojnicki, ,wörtlich genommen, besteht sie noch. Wir haben noch eine Armee (...). Aber sie zerfällt bei lebendigem Leibe. (...) Ein Greis, dem Tode geweiht, von jedem Schnupfen gefährdet, hält den alten Thron, einfach durch das Wunder, daß er auf ihm noch sitzen kann. (...) Die Zeit will uns nicht mehr! Diese Zeit will sich erst selbständige Nationalstaaten schaffen! Man glaubt nicht mehr an Gott. Die neue Religion ist der Nationalismus. Die Völker gehn nicht mehr in die Kirchen. Sie gehn in nationale Vereine. Die Monarchie (...) ist gegründet auf der Frömmigkeit: auf dem Glauben, daß Gott die Habsburger erwählt hat, über so und so viel christliche Völker zu regieren. (...) Der deutsche Kaiser regiert, wenn Gott ihn verläßt, immer noch; eventuell von der Gnade der Nation. Der Kaiser von Österreich-Ungarn darf nicht von Gott verlassen werden. Nun aber hat ihn Gott verlassen!"
\end{abstract}

Aus Roths Werken ist zugleich, auch aus dem hier zu untersuchenden Roman Radetzkymarsch, eine Sehnsucht nach einem Vaterland herauszuhören, ,nach dem Land des Vaters " ${ }^{\text {, }}$, wie Helmut Nürnberger in seiner Roth-Biographie bemerkt. ${ }^{11}$ David Bronsen schreibt in seiner Roth-Biographie, laut Bericht eines jüdischen Freundes von Roth soll er

${ }^{9}$ David B r o n s e n: Joseph Roth. Eine Biographie. München 1981, S. 28.

${ }^{10}$ Klaus N ü r n b e r g e r, Helmuth S c h r ö te r (Hrsg.): Joseph Roth. Reinbek 1981, S. 13.

${ }^{11}$ Roth ist ohne Vater aufgewachsen. Der Vater Nachum Roth, der aus Westgalizien stammte, verließ seine Mutter, nachdem er mit seinen privaten Geschäften in Hamburg und Kattowitz keinen Erfolg hatte. Er soll in dessen Folge in eine Anstalt für Geisteskranke eingeliefert worden und dort gestorben sein, ohne von der Existenz des Sohnes erfahren zu haben. Es liegt die Vermutung nahe, daß Roth diese autobiographische Episode in eine Szene im Radetzkymarsch transferiert hat. Es geht um den Besuch Herrn von Trottas bei dem wahnsinnig gewordenen Chojnicki nach dem Tod von Carl Joseph. Diesmal trauert der Vater um den verstorbenen Sohn, doch die Anspielung auf Roths Biographie, auf die Sehnsucht nach väterlicher Liebe, scheint die folgende Textpassage wiederzugeben: „Man sah Menschen mit irren Gesichtern und grausamen Verrenkungen der Gliedmaßen vorbeigehen, aber für den Bezirkshauptmann bedeutete Wahnsinn nichts Schreckliches, obwohl er zum erstenmal in einem Irrenhaus war. Schrecklich war nur der Tod. Schade! dachte Herr von Trotta. Wenn Carl Joseph verrückt geworden wäre, statt zu fallen, ich hätte ihn schon vernünftig gemacht. Und wenn ich es nicht gekonnt hätte, so wäre ich doch jeden Tàg zu ihm gekommen! Vielleicht hätte er den Arm so grauenhaft verrenkt wie dieser Leutnant hier (...). Aber es wäre doch sein Arm gewesen, und man kann auch einen verrenkten Arm streicheln. (...) Glücklich die Väter, deren Söhne verrückt sind!" (S. 240) Die angeführte Textpassage bringt nicht nur das Bedürfnis des Autors von Radetzkymarsch nach Liebe zum Ausdruck, sondern sie enthält auch seine Anklage gegen den Krieg, der die alte Optik und Werteordnung zerstört. In der Krisenzeit gilt wohl der Wahnsinn als die bessere Variante des Todes. 
in den Jahren der Emigration, die besonders schwer auf ihm lasteten und schon vernarbte Traumata wieder aufrissen, jeden Mitmenschen um seinen Vater beneiden. Roth, der in Wirklichkeit nie gewillt war, die Vaterschaft und deren Verantwortung auf sich zu nehmen, pflegte versonnen zu beteuern: „Wenn ich einen Sohn hätte, würde ich ihn nie aus den Augen lassen." 12

Einen Ersatz für den leiblichen Vater, den Roth nie kennengelernt hat, wird er in dem Staat Kaiser Franz Joseph I. finden: „Der Vater heißt Franz Joseph, der Erste. “" ${ }^{13}$ Die persönliche, ja fast intime Beziehung des Autors zum Kaiser und zur Donaumonarchie läßt auch folgende Textpassage erkennen: „Und weil der Tod des Kaisers meiner Kindheit genauso wie dem Vaterland ein Ende gemacht hatte, betrauerte ich den Kaiser und das Vaterland wie meine Kindheit." “14

Im Radetzkymarsch beschreibt der Autor den Prozeß des Untergangs seiner Kindheitswelt, der Donaumonarchie gegen Ende des Ersten Weltkriegs. Parallel zu dem historischen, literarisch erfaßten Geschehen wird der Verfall der Familie Trotta erzählt.

In Das Salz der Erde wird der Zerfall der Donaumonarchie aus der Perspektive des simplizianischen Huzulen Piotr Niewiadomski gesehen. Sein Name - Niemand, Unbekannt - verweist auf den Jedermanncharakter und die Anonymität dieser Gestalt im Vielvölkerstaat Galizien. Die Schnittstelle beider Romane, die zugleich als das den Mythos tragende und bildende Element anzusehen ist, stellt die umschwärmte und mit gottesähnlichen Zügen bedachte Gestalt des Kaisers dar:

Jeder Soldat schwor bei Gott, dem Allmächtigen, Kaiser Franz Joseph dem Ersten Treue. Er war eine Majestät von Gottes Gnaden, und er glaubte an Gott, den Allmächtigsten. Hinter dem goldbestirnten Blau des Himmels verbarg er sich, der Allmächtige - - - unvorstellbar! Seine Sterne waren es, die da am Himmel glänzten, und Sein Himmel war es, der sich über der Erde wölbte, und einen Teil der Erde, nämlich die österreichisch-ungarische Monarchie, hatte Er Franz Joseph dem Ersten zugeteilt. (S. 161f.) ${ }^{15}$

„In Das Salz der Erde vereinigte der Glaube an den Kaiser in diesen entlegenen Ländern die römischen Katholiken mit den griechischen Katholiken, die Armenier und die Juden zu einer gemeinsamen und allgemeinen Kirche." (S. 52) ${ }^{16}$

Die Gestalt des Kaisers überschattet menschliche Schicksale in den genannten Romanen und greift in sie ein. Im Radetzkymarsch wird der unaufhaltsame Zusammenbruch der Monarchie am Beispiel des über drei Generationen verlaufenden Zerfalls der kaisertreuen Familie von Trotta geschildert. Der Kaiser

${ }^{12}$ Aus einem Interview mit Soma Morgenstern. In: B r o n s e n (wie Anm. 9), S. 38.

${ }^{13}$ Joseph $\mathrm{R}$ o $\mathrm{t} \mathrm{h}$ : Rede über den alten Kaiser. In: Joseph $\mathrm{R}$ o $\mathrm{t} \mathrm{h}:$ Werke in drei Bänden. Bd. 3. Köln/Berlin. S. 406.

${ }^{14}$ Ebenda, S. 167.

15 Joseph Roth bekennt sich schon früh zur Monarchie, und den Kaiser-Mythos kreiert er nicht nur in seinen Werken, sondern er muß wohl selbst in dem Mythos aufgewachsen sein. Franz Werfel, der zu Roths Generation gehört, erinnert sich: „Sein Leben, sein Antlitz, seine gebrechlich elegante Altersfigur waren längst mythisch geworden. Sie erfüllten das Bewußtsein jedes Österreichers von dem Tag an, da er als sechsjähriges Kind die Volksschule zum ersten Mal betrat." Zitiert aus: B r o n s e n (wie Anm. 9), S. 103.

${ }^{16}$ Im folgenden wird zitiert aus Józef W i t 1 l i n : Das Salz der Erde. Leipzig/Weimar 1980. 
und die Idee der Monarchie erweisen sich in dem multikulturellen Land Galizien als die einzig verbindenden Elemente. Selbst innerhalb einer kleineren Gesellschaft der Familie von Trottas im Radetzkymarsch - werden die zwischenmenschlichen Beziehungen durch den Ehrenkodex (Großvater-Vater-Enkel-Beziehung) geregelt (vgl. S. 20f.).

Aus diesen kulturellen Unterschieden resultiert unverkennbar die Bewußtseinssowie Identitätskrise der Bewohner Galiziens, die sich sowohl in Das Salz der Erde als auch in Radetzkymarsch erspüren läßt.

In Radetzkymarsch, einem Generationenroman, in dem die drei Helden Repräsentanten ihrer Zeit sind, spielt die Gestalt des Kaisers eine gewichtige Rolle. Sie verbindet das Private der Familie mit dem Offiziellen der Monarchie und wird dadurch zum Kulminationspunkt. Das Verhältnis der Trottas - des Großvaters (des Helden von Solferino), seines Sohnes (des Bezirkshauptmanns Trotta) und des Enkelkindes Carl Joseph - zum Kaiser wird verschiedenartig gestaltet. Der Held von Solferino, Sohn eines slowenischen Bauern, hat dem Kaiser das Leben gerettet, wofür er den Adelstitel von Trotta erlangte. Doch weil sein Heldentum in einem Unterrichtsbuch zu einer kitschigen Tat umgedichtet wurde, verläßt er die Armee. Er zieht sich in ein ländliches, fast bäuerliches Leben zurück und verbietet seinem Sohn, Soldat zu werden. Dieser, ein Ideal des Beamten, dient dem Kaiser gewissenhaft, identifiziert sich mit der Monarchie und fühlt sich als Österreicher.

Er selbst, der Bezirkshauptmann, hatte niemals den Wunsch gespürt, die Heimat seiner Väter zu sehn. Er war ein Österreicher, Diener und Beamter der Habsburger, und seine Heimat war die Kaiserliche Burg zu Wien. (...) In seinem Bezirk vertrat er die Apostolische Majestät. Er trug den goldenen Kragen, den Krappenhut um den Degen. Er wünschte sich nicht, den Pflug über die slowenische Erde zu führen. In dem entscheidenden Brief an seinen Sohn stand der Satz: „Das Schicksal hat aus unserem Geschlecht von Grenzbauern Österreicher gemacht. Wir wollen es bleiben." (S. 93)

Carl Joseph von Trotta, Repräsentant der dritten Generation der Trottas, zeigt anfangs die gleiche Begeisterung für den Kaiser wie seine Vorfahren:

Er fühlte sich ein wenig den Habsburgern verwandt, deren Macht sein Vater noch hier repräsentierte und verteidigte und für die er einmal selbst ausziehen sollte, in den Krieg und in den Tod. Er kannte die Namen aller Mitglieder des Allerhöchsten Hauses. Er liebte sie alle aufrichtig, mit einem kindlich erhabenen Herzen, vor allen anderen den Kaiser, der gütig war und groß, der Armee besonders zugetan. Am leichtesten starb man für ihn bei Militärmusik, am leichtesten beim Radetzkymarsch. (S. 21)

Doch mit der Zeit werden ihm Österreich und der Kaiser immer fremder. Er identifiziert sich mit der Idee der Monarchie nur über die Gestalt des Großvaters. Der sich ankündigende Zerfall der Monarchie wird an Carl Josephs fortschreitendem Verlust der Bindung an den Kaiser und des Bezugs zur Donaumonarchie deutlich. Es ist zugleich ein Bruch in seiner Bindung an die Tradition, die sich aus dem Kult um den Kaiser speist, der vom Großvater und Vater betrieben wird. ${ }^{17}$

${ }^{17}$ Der in Radetzkymarsch von den drei Generationen von Trotta betriebene Kaiserkult und die von Generation zu Generation, von Vater (dem Bezirkshauptmann von Trotta) zu Sohn (Carl Joseph 
Carl Joseph, Freiherrn von Trotta, blieben die Tiere gleichgültig. Manchmal glaubte er, in sich das Blut der Ahnen zu fühlen: sie waren keine Ritter. (...) Der Vater des Großvaters war noch ein Bauer gewesen. Sipolje war der Name des Dorfes, aus dem sie stammten. Sipolje: das Wort hatte eine alte Bedeutung. Auch den heutigen Slowenen war es kaum noch bekannt. Carl Joseph aber glaubte es noch zu kennen, das Dorf. Er sah es, wenn er an das Porträt seines Großvaters dachte (...). Eingebettet lag es zwischen unbekannten Bergen, unter dem goldenen Glanz einer unbekannten Sonne, mit armseligen Hütten aus Lehm und Stroh. Ein schönes Dorf, ein gutes Dorf! Man hätte seine Offizierskarriere darum gegeben! Ach, man war kein Bauer, man war Baron und Leutnant bei den Ulanen! (...) Seitdem er zum Regiment eingerückt war, fühlte er sich als der Enkel seines Großvaters, nicht als der Sohn seines Vaters; der Sohn seines merkwürdigen Großvaters war er. (S. 45f.)

Doch die innere Schwäche Carl Josephs (er ist in eine Affäre um den Tod des Doktors Demant verwickelt), die auch die innere Situation der Donaumonarchie vor dem Ausbruch des Krieges symbolisierte, hat seine Entscheidung zur Folge, von den Ulanen zur Infanterie zu wechseln, was eine eindeutige Degradierung in der militärischen Karriere bedeutete. Die Peripherie der Monarchie, an die Carl Joseph versetzt wurde - vermutlich Roths Geburtsort -, wo „,keiner so kräftig ist wie der Sumpf, und wo (...) in der weltfernen, sumpfigen Öde der Garnison der oder jener Offizier der Verzweiflung, dem Hasardspiel, den Schulden und finstern Menschen verfiel“ (S. 97), impliziert eindeutig das Endstadium der glanzvollen Zeit der Donaumonarchie. Kriegs- wie auch Todesgefahr bestehen bereits: „Und keiner von den Offizieren des Zaren und keiner von den Offizieren der Apostolischen Majestät wußte um jene Zeit, daß über den gläsernen Kelchen, aus denen sie tranken, der Tod schon seine hageren, unsichtbaren Hände kreuzte." (S. 98) Dort verfällt Carl Joseph von Trotta dem Alkohol, macht Schulden. Sein Vater setzt sich für ihn beim Kaiser ein. Die Entscheidung, aus der Armee auszutreten, liegt bereits vor.

Doch immer wieder erinnert sich Carl Joseph von Trotta an den Kaiser, an seine Pflichten dem Monarchen gegenüber. Während des Festes der Dragoner, als die Gäste die Nachricht von der Ermordung des Thronfolgers erreicht und sie über den Kaiser und die Monarchie lästern, setzt er sich für den Kaiser ein.

„Ruhe!“” schrie Trotta, mit einer Stimme (...), vielleicht war es die Stimme des Helden von Solferino. Er fühlte sich eins mit dem Großvater. Er selbst war der Held von Solferino. Sein eigenes Bildnis war's, das unter dem Sulfit des väterlichen Herrenzimmers verdämmerte. (S. 222)

Carl Joseph verläßt die Armee. Auch sein Diener Onufrij zweifelt an der Zeit, an der Monarchie, an dem Sinn des Krieges und desertiert.

\footnotetext{
von Trotta) überlieferte dem Kaiser erwiesene Heldentat des Großvaters (des Helden von Solferino) scheinen feste Bestandteile des kommunikativen Gedächtnisses der Familie von Trotta zu sein. Dieses Gedächtnis wird mit ihrer Generation „sterben“. Jan Assmann nimmt zum kommunikativen Gedächtnis Stellung: „Das kommunikative Gedächtnis umfaßt Erinnerungen, die sich auf die rezente Zeit beziehen. Es sind die Erinnerungen, die der Mensch mit seinen Zeitgenossen teilt. Der typische Fall ist das Generationen-Gedächtnis. Dieses Gedächtnis wächst der Gruppe historisch zu; es entsteht in der Zeit und vergeht mit ihr, genauer mit seinen Trägern. Wenn die Träger, die es verkörperten, gestorben sind, weicht es einem neuen Gedächtnis. " A s s m a n n (wie Anm. 1), S. 50. - Der Autor reflektiert weiter, $\mathrm{da} ß$ es sich bei dem kommunikativen Gedächtnis um einen Erinnerungsraum handelt, der sich auf drei bis vier Generationen erstreckt. Vgl. ebenda. - In Radetzkymarsch bleibt die Erinnerung an die Zeit der Donaumonarchie und den Kaiserkult über drei Generationen hindurch bestehen.
} 
„Warum bist du desertiert?“ sagte Trotta. „Bin nur nach Hause gegangen!“ Es hatte keinen Sinn, so törichte Fragen zu stellen. Man verstand Onufrij gut. Er hatte dem Leutnant gedient, wie der Leutnant dem Kaiser. Es gab kein Vaterland mehr. Es zerbrach, es zersplitterte. (S. 229)

Dennoch empfindet Carl Joseph den Ausbruch des Krieges als einen erhabenen Augenblick, in dem sich sein von Kind an geträumter Traum, für den Kaiser zu sterben (S. 142), erfüllt. Es ist sein Krieg, seine Zeit, dem Kaiser einen treuen Dienst zu erweisen. Statt dessen stirbt der Enkel von Trotta während einer Schlacht zwar unter den Klängen des Radetzkymarschs, doch grotesk, fast banal: „Der Leutnant Trotta starb nicht mit der Waffe, sondern mit zwei Wassereimern in der Hand." (S. 237) Der Mythos einer heldenhaften, kaisertreuen Familie wird mit dem banalen Tod des Enkels des Helden von Solferino dekonstruiert. Auf den Tod Carl Josephs folgt im Roman der Tod des Kaisers:

Der Sohn war tot. Und auch der Kaiser starb. Und zum ersten Mal, seitdem Herr von Trotta die Todesnachricht erhalten hatte, glaubte er zu wissen, daß sein Sohn nicht zufällig gestorben war. Der Kaiser kann die Trottas nicht überleben! dachte der Bezirkshauptmann. Er kann sie nicht überleben! Sie haben ihn gerettet, und er überlebt die Trottas nicht. (S. 241)

Der Krieg, den man später den Ersten Weltkrieg nennen wird, bricht nun aus. Er wird im Roman symptomatisch durch Naturbilder angekündigt. ${ }^{18}$ Den hinterbliebenen Herrn von Trotta, der bislang Kaisertreue an den Tag legte, beschäftigt der Krieg weniger. „Und was ging ihn der Untergang der Welt an, den er jetzt noch deutlicher sah, als einstmals der prophetische Chojnicki? Sein Sohn war tot. Sein Amt war beendet. Seine Welt war untergegangen. “(S. 238) Er stirbt kurz nach dem Ausbruch des Krieges. Der symbolische Tod des Barons von Trotta, der dem Kaiser sein Leben unterordnete und die Idee der Monarchie zu seiner beruflichen und existentiellen Pflicht erhoben hatte, mutet an wie eine Absage an die ungewisse Zeit und neue Weltordnung. Es scheint, daß Radetzkymarsch als ein Ausschnitt aus dem „Weltuntergang“, den Joseph Roth im Zusammenhang mit dem Zerfall der Monarchie erlebt hat, anzusehen ist. ${ }^{19}$ In dem Gefühl heimatlos zu sein, wurzelt wohl der Hang des Autors, das Vergangene durch Erinnerung zu rekonstruieren und aufgrund eigener Erfahrung der Vergangenheit zu mythisieren.

Ähnlich wie bei Roth prägten zwei Schlüsselerfahrungen Wittlins schriftstellerisches Schaffen: der Zerfall der österreichisch-ungarischen Monarchie und damit der verbundene Verlust des „Vaterlandes auf Zeit“, das „mit dem Leben eines jeden Sohnes“ untergeht, sowie der Erste Weltkrieg, der Völkermord. ${ }^{20}$ Das

${ }^{18}$ Die Gäste auf dem Fest der Dragoner erfahren von der Ermordung des Thronfolgers vor einem heftigen Gewitter. Dann zeigen sich Raben als Todes- und Kriegsboten. Der heisere Schrei der Gänse und die Glocken über den Städten verkünden ebenfalls den Krieg.

${ }_{19}^{19}$ Den Heimatverlust artikuliert Roth folgendermaßen: „Schwaby gibt es nicht mehr. Alles was ich betrete, geht unter." Aus: B r o n s e n (wie Anm. 9), S. 29, und: „Wir sind alle Bruchstücke, weil wir die Heimat verloren haben." (Ebenda, S. 28).

${ }^{20}$ Vergleiche dazu Hubert O r ło w s k i : Pazifismus und Kakaniens Untergang im Schaffen von Józef Wittlin. In: Klaus A m a n n, Hubert L e n g a u e r (Hrsg.): Österreich und der große Krieg 1914-1918. Die andere Seite der Geschichte. Wien 1989, S. 262. 
Gefühl der Heimatlosigkeit und die Erfahrung des Verlustes tauchen, ähnlich wie bei Roth, auch bei Wittlin auf:

Ich hatte schon in meiner Vorstellung den Untergang großer Hauptstädte Europas gesehen, ich hatte die schönsten Orte in der Welt gesehen, die zerstört, zerbombt und vergast waren. Alles nämlich, was in den letzten Jahren in der Welt angerichtet wurde, war mehr oder weniger eine vorgeplante Entwicklung der Vernichtung. Ich bereiste Orte und Landschaften, die ich von früheren Reisen kannte, doch ich sollte auch viele Städte zum ersten Mal in meinem Leben sehen. Ich fuhr ihnen entgegen, um sie zu begrüßen und zugleich zu verabschieden. Jenseits des Grabes, mit einem Totenlicht haben die entfernten Städte wie Paris, Marslilien, weiße Städtchen der Provence phosphorisiert (...) In jedem Ort des heutigen Europas sind wir Gräber unserer selbst, düstere Verurteilte, mit der Sünde gegen die Hoffnung belastet. ${ }^{21}$

Seine Autobiographie und den Verlust des „Vaterlandes auf Zeit ${ }^{\text {‘22 }}$, wird Wittlin in Das Salz der Erde in Erinnerung bringen, indem er den ÜbervaterMythos des Kaisers Franz Joseph ${ }^{23}$ gestaltet sowie die idyllische Welt Ostgaliziens der Krisenstimmung vor dem Ausbruch des Ersten Weltkriegs gegenüberstellt. Die Naturschilderung scheint den Untergang der alten Weltordnung symbolisch unverkennbar wiederzugeben:

Die alte Großmutter Donau rauschte hinter dem Prater, wie gewöhnlich, und trug ihre ewige Ruhe ins Schwarze Meer, um sie dort gleichsam zu bewahren. Ihre letzte Welle nahm für ewig die bezaubernde Melodie dieser Stadt mit, eine Melodie, die nie mehr zurückkehren wird. (S. 20)

Der Name des Helden in Wittlins Roman, Niewiadomski, verdeutlicht die Entindividualisierung und Einverleibung der namen- und geschichtslosen Individuen in das Kaiserreich:

Peter war auch ein Kaiserlicher. Niemals verließ ihn das Bewußtsein, wem er diene, scheinbar nur schleppte er Lasten für Gutsbesitzer, scheinbar bediente er Juden, die in seiner Gegend mit Getreide und Kartoffeln handelten. In Wirklichkeit tat er das alles für den Kaiser. Dafür bezahlte ihn der Kaiser und schützte ihn durch besondere Gesetze. (S. 39)

Peters Existenz im Vielvölkerstaat Galizien scheint daher die eines Untertans zu sein, geprägt von der Pflicht, an Kaiser und Gott zu glauben. (S. 161) In diesem Glauben an den österreichisch-ungarischen Kaiser liegen Peter Niewiadomskis Lebenserwartungen. Er will für sein Leben gern vom Hilfsarbeiter auf der Station Topory zum selbständigen Posten eines Bahnwärters aufsteigen. Dieser soziale Aufstieg ermöglicht es ihm, die Amtsmütze mit dem kaiserlichen Zeichen zu

${ }^{21}$ Wittlin, Józef: Orfeusz w piekle XX wieku. Paris 1963, S. 252.

${ }^{22}$ Ebenda.

${ }^{23}$ Es entsteht der Eindruck, daß Das Salz der Erde die Funktion eines Mythos erfültt. Der Roman schildert nicht eine Welt, sondern er legt sie aus. Zur Problematik des Mythos als einer Kategorie in der Literaturwissenschaft siehe z.B.: E. K u ź m a : Kategoria mitu $w$ badaniach literackich. In: Pamiętnik Literacki, 1986, H. 4 und H. M a r k i e w i c z: Literatura a mity. In: Twórczość, 1987, H. 10. - Peter Niewiadomskis Weltvorstellung, die im christlichen Glauben tief verankert ist, wird dem wahren Weltbild gegenübergestellt - dem bevorstehenden Krieg -, der den Protagonisten befremdet. Niewiadomski mythisiert somit die Welt, der sich nähernde Krieg entzaubert hingegen die von ihm wahrgenommene Idylle. 
tragen. Auch in das Schicksal dieses Protagonisten greift der ferne, von Peter mythisierte Kaiser ein. Peters Wunsch, Bahnwärter zu werden, geht mit dem Ausbruch des Krieges in Erfüllung. Den Krieg empfindet Niewiadomski anfangs nicht als eine Gefahr oder als ein Anzeichen für den Untergang des kaiserlichen Imperiums, sondern lediglich als Änderung im Eisenbahnverkehr. (S. 46) Statt der gewohnten Personenzüge fahren jetzt an Topory-Czernielitza militärische Transporte vorbei. Der Bahnhofsvorsteher beauftragt Peter, die Plakate mit der Kaiserrede „An meine Völker“ im Warteraum der Station Topory-Czernielitza anzubringen. Grotesk und banal mutet diese Szene an, weil der Analphabet Niewiadomski „das kaiserliche Manifest verkehrt angebracht hat“. (S. 51) Der Protagonist zeigt sich wohl nicht imstande, die Idee der Monarchie zu tragen, weil er nicht lesen und schreiben kann sowie kein „Nationalbewußtsein“ hat. (S. 161) Sein bislang durch nichts gestörtes Leben, er hat ein Haus, die Geliebte Magda und einen Hund, ändert sich mit der kaiserlichen Einberufung zum Militär. Er zeigt sich vor der Musterungskommission in der Stadt Śniatyń gelassen. Erst der Anblick der Menschen verschiedener Herkunft und Berufe im Warteraum der Kommission bringt ihn auf Gedanken, die das Jüngste Gericht heraufbeschwören:

\begin{abstract}
So wird voraussichtlich der Wartesaal des Jüngsten Gerichts aussehen, wo sämtliche Unterschiede aufgehoben werden, die einst Menschen trennten. Hier waren alle Menschen nackt und der Gestank, dieser repräsentative Geruch des irdischen Seins, war das irdische Element. Jeder Körper brachte Düfte seines Hauses mit sich, das Aroma seiner alltäglichen Mühe, wie auch die Ausdünstungen der Tiere, die mit ihm diese Mühe teilten. Huzulen brachten die stickige Luft ihrer elenden, nie gelüfteten Lehmhütten mit, die Ackerleute rochen noch nach Erde und Getreide, die Hirten nach Dünger, die Juden - nach Schenke, Mühle und Schabbes. (S. 76)
\end{abstract}

Die Hände der Menschen im Wartezimmer sehen für den Erzähler wie „Werkzeuge des Mordes“ aus. (S. 79) Hier wird entschieden, wer für den Krieg taugt und einen militärischen, ehrenvollen Tod und wer einen zivilen - als „Folge häuslicher Leiden und Gebrechen " - finden darf. (S. 83)

Der Tod des Papstes stand für den gottesfürchtigen Peter in einem engen Zusammenhang mit dem heraufziehenden Krieg:

Wahrscheinlich starb der Heilige Vater deswegen (...), weil die Geistlichen das Töten gestatten. Sie haben es nicht nur erlaubt, sondern sie haben es auch ausdrücklich empfohlen. Es sieht beinahe so aus, als ob das Töten von Russen keine Sünde sei oder als ob es als Halbsünde angerechnet werde wie das Töten eines Juden. (S. 160) ${ }^{24}$

Niewiadomski erfüllt der Krieg nicht mit Angst und das Ziehen in den Krieg nicht mit dem Gefühl des Verlustes oder der Heimatlosigkeit. Denn Peter als ein „Kaiserlicher“ hat nicht viel zu verlieren. Er selbst versteht sich als dessen Eigentum. Die Gestalt des Kaisers wird hier mythisiert; sie bekommt göttliche Züge - der Kaiser gibt und nimmt:

${ }^{24}$ Es scheint, daß sich Wittlin an dieser Stelle als Antikriegsautor zu Wort meldet. Für den Schriftsteller „,ist jeder Krieg auf der Welt ein Bruderkrieg“. Aus: Józef W i t $\mathrm{l}$ i n : Die Geschichte vom geduldigen Infanteristen. Frankfurt (M.) 1986, S. 387. 
Alles auf dieser Erde ist des Kaisers oder Gottes Eigentum, dachte Peter. Die Erde und der Himmel, der Prut und der Czermosz, die Karpaten und die Kühe, Hunde und Menschen gehören Gott. Die ganze Eisenbahn dagegen - alle Waggons und Lokomotiven, alle Wärterhäuschen und Schranken, sogar ein Stück verrosteten Drahts (...) - gehört dem Kaiser. Wer eine Schwell stiehlt, schädigt den Kaiser, und dafür nimmt ihn ein kaiserlicher Gendarm ins kaiserliche Gefängnis. Recht hat er! (...) Und wem gehört das Geld? Es gehört dem, dessen Haupt daraufgeprägt ist. Der Kaiser gibt dem Menschen Geld, so wie ihm Gott das Leben gibt. Das eine aber wie das andere sind Leihgaben. Der Kaiser macht mit Gott verschiedene Geschäfte, deswegen hat er das Recht, dem Menschen das Leben zu nehmen, das ihm Gott geboren hat. (S. 68)

Die Mobilisation bedeutet für Niewiadomski daher den Ruf des Kaisers, dem er selbstverständlich folgen wird. Sein gewohntes Leben, seine Dienstmütze muß er nun gegen eine Uniform und das Soldatenleben im ungarischen Ausbildungslager tauschen. Dem Multikulturellen, das ihn befremdet, das für ihn das Teuflische versinnbildlicht und bei ihm Angst auslöst und überhaupt nicht zum gemeinsamen Kampf für die Monarchie inspiriert, begegnet Peter während der Fahrt nach Ungarn:

Plötzlich wurde sich Peter darüber klar, was für eine Sünde der Turmbau zu Babel gewesen sein mußte, wenn Gott darum die menschlichen Zungen vermischte. Peter hatte nie vorher über die Ausmaße dieser Katastrophe nachgesonnen (...), warum ein Mensch den anderen nicht versteht. (...) Und wäre es wenigstens der Feind, ein Russe, Serbe, aber das ist sozusagen einer von den unseren, ein Kaiserlicher ... Nein, das war kein kaiserlicher Mensch. Ein kaiserlicher Mensch wird den anderen kaiserlichen Menschen immer irgendwie verstehen, und sei es auf deutsch. Dieser Gendarm ist - ist ein königlicher Mensch. Weil der Kaiser bei den Magyaren nur ein König ist. Da sieht man's - die Magyaren verdienen nicht mal einen Kaiser. Mit so einem Maul und so einer Sprache. Was will der Teufel eigentlich? (...) Die Kaiserlichen hörten nicht auf den Gendarmen. Sie leisteten passiven Widerstand, waren sie doch in unbedingter Mehrheit ... (S. 198f.)

Dem Kaiser treu, duldet Niewiadomski jeden Mißstand, jede Ungerechtigkeit, die er dort vorfindet, beispielsweise den Mißbrauch, den Bachmatiuk gegenüber den verängstigten Rekruten treibt, und die das Gesetz der Subordination zuläßt und rechtfertigt. Der Preis für die Subordination ist, ein echter Soldat und dadurch erst Mensch zu werden:

Gott schuf die Welt in sechs Tagen und brauchte seine Ruhe, und Unteroffiziere müssen wochenlang Menschen zu Soldaten, also zu wirklichen Menschen umarbeiten. Denn der von Gott geschaffene Mensch (...) ist kaum Material für einen Menschen, kaum Halbrohstoff.

Der Mythos einer heilen Welt, den Peter Niewiadomskis Weltanschauung aufzeigt, die Harmonie der Welt gesichert durch den Glauben an den Kaiser, wird dem Bild der mit dem Krieg zu Ende gehenden alten Ordnung gegenübergestellt. Unruhen im Ausbildungslager, Angstzustände der Soldaten wegen des nahenden Krieges, Vorbereitungen auf den Kampf schaffen die Wirklichkeit am Vorabend des Ersten Weltkrieges. Die Wirklichkeit, die in Das Salz der Erde aufscheint, bildet die Geschichte des Krieges ,,vor dem Kriege, vor den Kriegshandlungen, vor den Handlungen, die mit Töten und Getötetwerden gleichgesetzt werden müssen “ ${ }^{25}$

${ }^{25}$ Siehe Orłows ki (wie Anm. 20), S. 264. 
Das tragische Schicksal der galizischen Bevölkerung, der kaisertreuen Bauern, die für die Idee der Monarchie sterben müssen, klingt hier an: „Die Huzulinnen-Mütter neideten den Mutter-Kühen die selige Unkenntnis des Schicksals ihrer Nachkommenschaft, die in Schlachthäusern gemordet wurde." (S. 113)

Im Ausbildungslager nimmt Niewiadomski die neue Ordnung, vor allem seine eigene Funktion im Kriegsgetriebe, verwirrt wahr:

Er konnte die neue Ordnung der Dinge nicht fassen. Einundvierzig Jahre lebte er nun schon auf der Welt, und immer lernte er sie neu kennen und immer von einer neuen Seite. Von einer schlimmeren. Gut, sagte er sich, es ist Krieg. Das wissen wir. Aber warum häuft der Kaiser soviel Angst, soviel Wut, so viele Strafen gegen seine eigenen Menschen? Wäre es nicht besser, die ganze Wut für den Russen aufzuheben? Man führt doch mit ihm den Krieg und nicht mit uns. Wozu verdirbt man österreichisches, gutes, katholisches Blut? (S. 254f.)

Es scheint, daß sich hinter diesen „Kriegsbildern vor dem Krieg“26 die Poetik des Werkes verbirgt. Durch Peters grotesk anmutende Wahrnehmung der Welt wird einerseits der Mythos Galizien und der gottähnlichen Gestalt des Kaisers gebildet und andererseits dekonstruiert. Im Nachwort zu Wittlins Roman schrieb Peter Härtling:

Dieses Buch über den Krieg ist nicht zu vergleichen mit anderen Büchern über den Krieg. Es spricht nicht für den Krieg, es erzählt über den Anfang des Krieges, des Ersten Weltkrieges, vom Anfang der Angst (...). Im Anfang steckt hier schon das Ende: die Verwüstung und die brutale Anonymität, aus der sich die Erinnerungen nicht mehr befreien. ${ }^{27}$

Was verbindet jedoch die beiden so unterschiedlichen Romane Radetzkymarsch und Das Salz der Erde? Es sind zweifelsohne die thematische und temporale Dimension sowie Galizien als Schauplatz des Zerfalls der Donaumonarchie. Die Romane verbindet ideologisch ebenfalls die Identitätskrise der in den Werken agierenden Helden. Auf den gemeinsamen Nenner des deutschpolnischen Gedächtnisraums Galizien bringt sie schließlich der poetologische Aspekt - die Mythologisierung. Das Kronland Galizien wird somit zu einer von Mythen umwobenen Landschaft und die Gestalt des Kaisers zur mythischen Figur zum Innbegriff der Güte und der Gerechtigkeit. So in der Literatur aufgehoben, leben sie fort und erleben ihre Blütezeit immer aufs Neue.

${ }^{26}$ Ebenda, S. 265.

${ }^{27}$ Peter H ä r $\mathrm{l}$ i n g im Nachwort zur Neuausgabe des Romans bei Fischer, Frankfurt (M.) 1984, S. 313. 